\title{
Comparison of Glove Specifications, 3D Hand Scans, and Sizing of Sports Gloves for Female Athletes
}

\author{
Linsey A. GRIFFIN¹, Susan SOKOLOWSKI ${ }^{2}$, Elisheva SAVVATEEV ${ }^{1}$, \\ Arif-UI-Anwar BHUYAN ${ }^{1}$, Nathan ROESE ${ }^{2}$ \\ ${ }^{1}$ University of Minnesota, St Paul MN, USA; \\ 2 University of Oregon, Portland OR, USA \\ https://doi.org/10.15221/19.109
}

\begin{abstract}
Gloves are used in many sports as a form of protective gear and to enhance performance. Inadequately fit gloves can be detrimental to an athlete's ability to perform. As the population of female athletes grows and diversifies, it is important to ensure glove fit and performance across the plethora of sports. Despite improvements in the availability of anthropometric data, measurements of the hand remain limited. In recent years, 3D scanning has improved to capture complexities of the hand. 3D scan data of the hand offers potential to improve the sizing, fit and design of gloves for sports.

There is a need to better understand anthropometric hand data in relation to female athletes and sports activities to improve future glove fit standards and performance. The purpose of this study was to collect, compare, and analyze 3D hand scans ( 30 subjects) versus actual glove specifications and sizing of commonly used sports gloves. A close fitting golf glove (FootJoy Women's StaSof) was selected for comparison. Scans were taken of each subject's dominant hand (landmarked in 30 locations for accuracy) with the Occiptial Structure Sensor and iPad, and measured with Anthroscan software. Detailed specifications of the gloves that related to measurements of the hand were recorded and compared to the population measurements to determine how well the selected glove model fit each subject. To better serve diverse users, results suggest that traditional glove sizing is inadequate and more anthropometric data of the hand are needed to inform better glove sizing and fit. Based upon the findings of this study, future studies will be conducted to evaluate various glove brands and activities.
\end{abstract}

Keywords: Athletic gloves, sports, sizing, 3D scanning, anthropometric measures, fit

\section{Introduction}

Gloves are used in many sports as a form of protective gear and to enhance performance. Inadequately fit gloves can be detrimental to an athlete's ability to perform. As the population of female athletes grows and diversifies, it's important to ensure glove fit and performance across the plethora of sports. Close fitting gloves are used in numerous sports ranging from golf to softball. Glove design and fit are traditionally created using limited anthropometric data. Despite improvements in the availability of anthropometric data, measurements of the hand remain limited. In recent years, 3D scanning has improved to capture complexities of the hand. 3D scan data of the hand offers potential to improve the sizing, fit and design of gloves for sports.

There is a need to better understand anthropometric hand data in relation to female athletes and sports activities in order to improve future glove fit standards and performance. For this research, golf and golf gloves were selected to examine the relationship between female hand anthropometry and glove specifications. This pilot study will explore state-of-the-art leather golf glove sizing, specifications across a size range, along with how well 3D female hand scans size and fit into a leading U.S. brand.

\section{Background}

\subsection{Women in Golf and Definition of golf sports gloves}

The modern sport of golf has origins in $15^{\text {th }}$ Century Scotland, and continues to attract players worldwide with skill sets ranging from hobby to professional. Today, the percentage of women playing golf is around $24 \%$ and the percentage of women entering the sport is increasing every year [1].

Gloves are worn by players to improve grip on the golf club as a player swings (figure 1). A golf glove can provide a tackier grip, more friction on the club, and can prevent the club from turning in a player's hand. Gloves are traditionally worn on one hand, however, some players wear them on both hands. 
Leather golf gloves covering the hand and wrist are the most common glove, however gloves with synthetic materials or a combination of materials are increasing their market share. Because of the nature of how golf gloves are used, a next-to-skin fit is ideal. Incorrect fit, such as any extra material in the fingers and/or palm region, can have detrimental effects on a player's game.

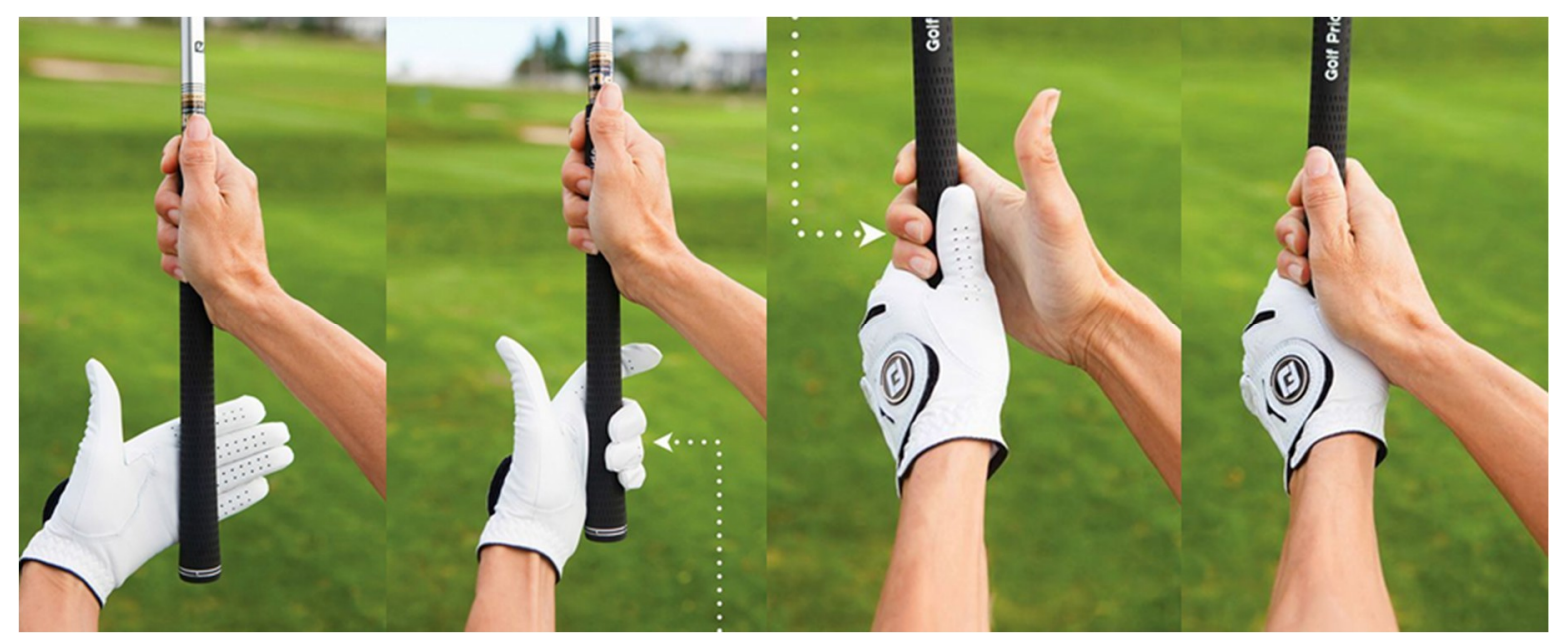

Fig. 1. Image of hands grasping club with golf gloves [2]

\subsection{D Anthropometric hand data of women}

Glove fit challenges exist because there is a lack of accurate and relevant anthropometric data for designers [3]. This is especially true for women, as the majority of anthropometric data collected in the U.S. is from military personnel, includes limited measures (e.g., hand circumference, breadth, and Length), and is only in a 2D format. To develop better performing products for the hand, the researchers are interested in building a more accessible 3D women's hand scan database and improving the relationship of the 3D scan data to product design. To date, the researchers have investigated methodology to collecting 3D hand scan data, 3D hand scan technology assessment, hand proportions as it relates to designing gloves from a sketch, dynamic hand scanning and a performance glove design process [4-12].

\section{Experimental Procedures}

Having gloves that properly fit women is imperative to comfort and athletic performance. The relationship of the measurements of the hand and the measurements of a glove are a key factor in providing good fit to the wearer. The purpose of this research was to pilot a method to collect, compare, and analyze 3D hand scans of women to the specifications of a commonly sold leather golf glove purchased in the U.S.

\subsection{D Hand scanning method}

A convenience sample of thirty women were recruited from the University of Minnesota and the University of Oregon. Prior to scanning, subjects reviewed and signed a consent form approved by the Institutional Review Board of the partnering institutions. Each subject's dominant hand was landmarked using washable marker and vinyl circular stickers at twenty-six locations. The landmark locations are based on the procedure outlined by Griffin, Kim, Carufel, Sokolowski, Lee, and Seifert [6].

Next, the subject's hand was 3D scanned in a splayed position using an Occipital Structure Sensor attached to an Apple iPad. The splayed hand position was selected based on ISO 7250-1:2017 method for collecting anthropometric measurements and the ease of measurement comparison between the selected glove and the 3D scan [13]. A frame with clear plexiglass was used to stabilize the hand during the scanning process which took approximately one-minute per scan. Figure 2 shows the scanning process using the Occipital Structure Sensor and an example of the resulting scans. The scans with color and texture were saved as .OBJ files, cleaned and oriented in the AnthroScan software. 

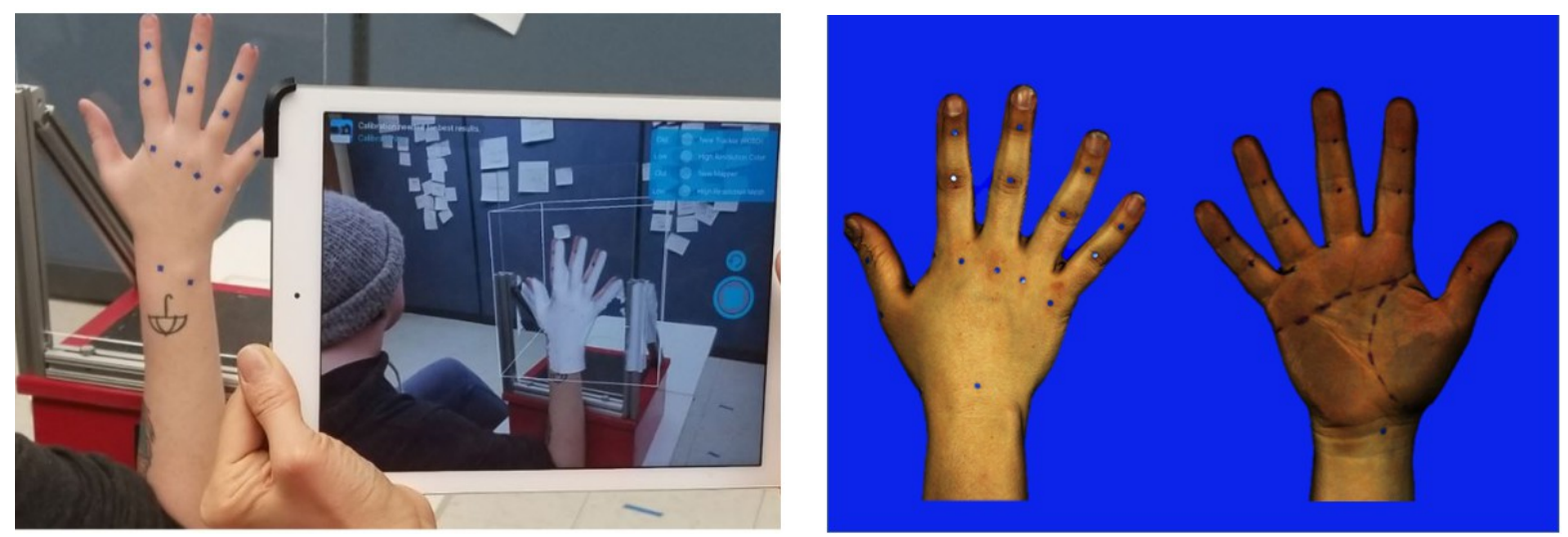

Fig. 2. 3D hand scanning process with Occipital Structure sensor

\subsection{Glove selection and justification}

The two leading golf equipment companies are Acushnet, who own Titleist and FootJoy, and Callaway [14]. In 2018, FootJoy sold $\$ 439.68$ million in gear, Callaway sold 329.89 million, and Titleist 146.07 million [14]. Based on this data, FootJoy is likely to have the largest market share in gloves, followed by Callaway and Titleist.

The glove selected for this study was the FootJoy Women's StaSof golf glove. This traditional women's golf glove is constructed from an exclusive leather from Pittards of England that is designed to optimize and retain softness and flexibility [15]. The glove design features a negative cut with Keystone thumb, secure closure on the back of the hand, elastic on the back of the hand and wrist, and strategically placed perforation to improve breathability and flexibility. A negative cut in gloves is a style of cut that has finger gussets between the palm and backhand, which allows the fingers to be held in place and produces an overall snugger fit. A keystone thumb style features a set-in thumb piece and is known for comfort and improved dexterity in glove design.

The FootJoy StaSof glove comes in four women's sizes: Small (LS), Medium (LM), Medium-Large $(\mathrm{LML})$, and Large (LL). This model of glove was selected because FootJoy has the largest glove marketshare, they market multiple sizes for women, the product is available to women at retail and online, and the glove has extensive size selection procedures provided by the manufacturer.
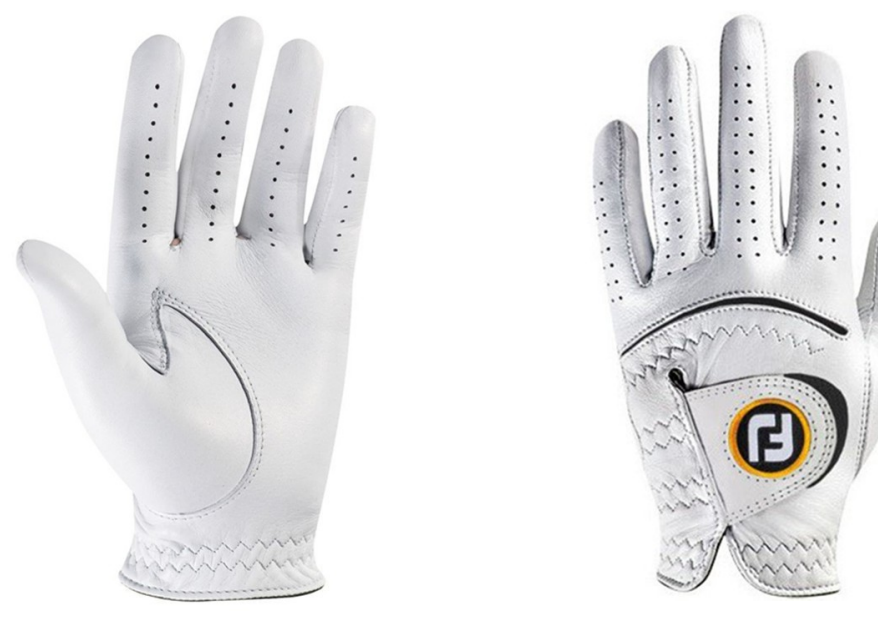

Fig. 3. FootJoy StaSof Leather Womens Golf gloves

\subsection{Measurement definitions}

Once the 3D scans were obtained and the gloves selected, measurements were defined to enable an accurate comparison between the two. Traditionally, anthropometric measurements and product measurements vary greatly, and it is not possible to do a direct comparison between manufacturer specifications and an anthropometric database. The value of 3D scans is that they enable researchers to take an infinite number of measurements that are product specific. For this study, the researchers 
defined fourteen measurements that are critical to glove fit that included a range of finger and hand lengths, widths, and circumferences. The palm circumference measurements were defined based on common anthropometric measurements and the manufacturer defined method for size selection. Figure 4 provides a diagram of the comparative hand and glove measurement locations. Table 1 provides definitions and locations of each hand and glove measurement and delineates the measurement type (surface or linear).
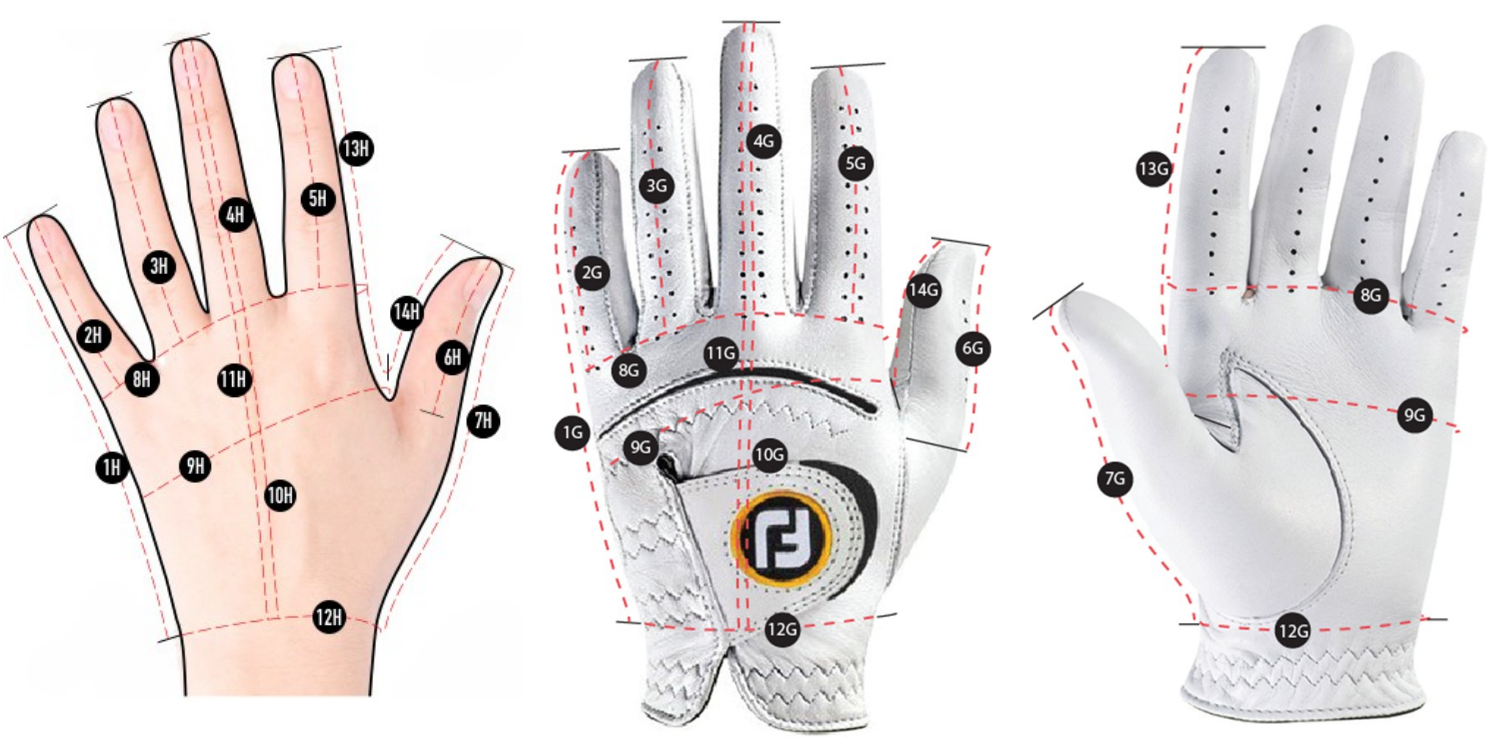

Fig. 4. Measurement points for hand and gloves

Table 1. Comparative hand and glove measurement definitions and locations.

\begin{tabular}{|l|l|}
\hline $\begin{array}{l}\text { Hand } \\
\text { Measure \# }\end{array}$ & $\begin{array}{l}\text { Measurement } \\
\text { Definition }\end{array}$ \\
\hline $1 \mathrm{H}$ & $\begin{array}{l}\text { Length measure from the distal tip of } 5^{\text {th }} \\
\text { finger to wrist crease. Measure follows } \\
\text { along the side of the hand. Surface } \\
\text { measure. }\end{array}$ \\
\hline $2 \mathrm{H}$ & $\begin{array}{l}\text { Length measure from the distal tip of } 5^{\text {th }} \\
\text { finger to the interdigital fold. Measure is } \\
\text { centralized through the finger. Linear } \\
\text { measure. }\end{array}$ \\
\hline $3 \mathrm{H}$ & $\begin{array}{l}\text { Length measure from the distal tip of } 4^{\text {th }} \\
\text { finger to the interdigital fold. Measure is } \\
\text { centralized through the finger. Linear } \\
\text { measure. }\end{array}$ \\
\hline $4 \mathrm{H}$ & $\begin{array}{l}\text { Length measure from the distal tip of } 3^{\text {rd }} \\
\text { finger to the interdigital fold. Measure is } \\
\text { centralized through the finger. Linear } \\
\text { measure. }\end{array}$ \\
\hline $5 \mathrm{H}$ & $\begin{array}{l}\text { Length measure from the distal tip of } \\
\text { 2nd finger to the interdigital fold. } \\
\text { Measure is centralized through the } \\
\text { finger. Linear measure. }\end{array}$ \\
\hline $6 \mathrm{H}$ & $\begin{array}{l}\text { Length measure from the distal tip of } 1 \mathrm{st} \\
\text { finger to the interdigital fold. Measure is } \\
\text { centralized through the finger. Linear } \\
\text { measure. }\end{array}$ \\
\hline
\end{tabular}

\begin{tabular}{|l|l|}
\hline $\begin{array}{l}\text { Glove } \\
\text { Measure \# }\end{array}$ & $\begin{array}{l}\text { Measurement } \\
\text { Definition }\end{array}$ \\
\hline $1 G$ & $\begin{array}{l}\text { Length measure from the distal tip of } \\
\text { glove's } 5^{\text {th }} \text { finger to the glove's wrist. } \\
\text { Measure follows along the side of } \\
\text { the glove. Surface measure. }\end{array}$ \\
\hline $2 G$ & $\begin{array}{l}\text { Length measure from the distal tip of } \\
\text { glove's } 5^{\text {th }} \text { finger to the lower finger } \\
\text { crotch. Measure is centralized } \\
\text { through the finger. Linear measure. }\end{array}$ \\
\hline $3 G$ & $\begin{array}{l}\text { Length measure from the distal tip of } \\
\text { glove's } 4^{\text {th }} \text { finger to the lower finger } \\
\text { crotch. Measure is centralized } \\
\text { through the finger. Linear measure. }\end{array}$ \\
\hline $4 G$ & $\begin{array}{l}\text { Length measure from the distal tip of } \\
\text { glove's } 3^{\text {rd }} \text { finger to the lower finger } \\
\text { crotch. Measure is centralized } \\
\text { through the finger. Linear measure. }\end{array}$ \\
\hline $5 G$ & $\begin{array}{l}\text { Length measure from the distal tip of } \\
\text { glove's } 2^{\text {nd }} \text { finger to the lower finger } \\
\text { crotch. Measure is centralized } \\
\text { through the finger. Linear measure. }\end{array}$ \\
\hline $6 G$ & $\begin{array}{l}\text { Length measure from the distal tip of } \\
\text { glove's 1st finger to the crotch. } \\
\text { Measure runs parallel to the fold of } \\
\text { the thumb pattern. Linear measure. }\end{array}$ \\
\hline
\end{tabular}




\begin{tabular}{|l|l|}
\hline $7 \mathrm{H}$ & $\begin{array}{l}\text { Length measure from the distal tip of } 1^{\text {st }} \\
\text { finger to wrist crease. Measure follows } \\
\text { along the side of the hand. Surface } \\
\text { measure. }\end{array}$ \\
\hline $8 \mathrm{H}$ & $\begin{array}{l}\text { Circumferential measure at the } 2^{\text {nd }} \text { to } 5^{\text {th }} \\
\text { finger interdigital folds. Surface } \\
\text { measure. }\end{array}$ \\
\hline $9 \mathrm{H}$ & $\begin{array}{l}\text { Circumferential measure at the bottom } \\
\text { of the } 1 \text { st finger interdigital fold parallel } \\
\text { to } 8 \mathrm{H} \text { or } 12 \mathrm{H} \text {. Surface measure. }\end{array}$ \\
\hline $10 \mathrm{H}$ & $\begin{array}{l}\text { Length measure from the } 3^{\text {rd }} \text { finger } \\
\text { interdigital fold to the wrist. Measure is } \\
\text { centralized through the } 3^{\text {rd }} \text { metacarpal. } \\
\text { Linear measure. }\end{array}$ \\
\hline $11 \mathrm{H}$ & $\begin{array}{l}\text { Length measure from the distal tip of } 3^{\text {rd }} \\
\text { finger to the wrist. Measure is } \\
\text { centralized through the } 3^{\text {rd }} \\
\text { Linear meacarpal. }\end{array}$ \\
\hline $14 \mathrm{H}$ & $\begin{array}{l}\text { Circumference measure of the smallest } \\
\text { part of the wrist. Surface measure. }\end{array}$ \\
\hline $12 \mathrm{H}$ & $\begin{array}{l}\text { Length measure from the distal tip of } 2^{\text {nd }} \\
\text { to distal tip of } 1^{\text {st }} \text { finger. Surface } \\
\text { measure. }\end{array}$ \\
\hline $13 \mathrm{H}$ & $\begin{array}{l}\text { finger to the interdigital fold. Surface } \\
\text { meare. }\end{array}$ \\
\hline
\end{tabular}

\begin{tabular}{|l|l|}
\hline $7 G$ & $\begin{array}{l}\text { Length measure from the distal tip of } \\
\text { glove's 1st finger to the glove's wrist. } \\
\text { Measure follows along the side of } \\
\text { the glove. Surface measure. }\end{array}$ \\
\hline $8 G$ & $\begin{array}{l}\text { Circumferential measure around the } \\
\text { glove's } 2^{\text {nd }} \text { to } 4^{\text {th }} \text { lower finger } \\
\text { crotches. Surface measure. }\end{array}$ \\
\hline $9 G$ & $\begin{array}{l}\text { Circumferential measure at the top } \\
\text { of the 1st finger glove's crotch } \\
\text { parallel to the wrist. Surface } \\
\text { measure. }\end{array}$ \\
\hline $10 G$ & $\begin{array}{l}\text { Length measure from the glove's } 3^{\text {rd }} \\
\text { finger crotch to the glove's wrist. } \\
\text { Measure is squared through the } \\
\text { backhand of the glove. Linear } \\
\text { measure. }\end{array}$ \\
\hline $11 G$ & $\begin{array}{l}\text { Length measure from the distal tip of } \\
\text { glove's } 3^{\text {rd }} \text { finger to the glove's wrist. } \\
\text { Measure is squared to the backhand } \\
\text { of the glove. Linear measure. }\end{array}$ \\
\hline $12 G$ & $\begin{array}{l}\text { Circumference measure of the } \\
\text { smallest part of the glove's wrist. } \\
\text { Surface measure. }\end{array}$ \\
\hline $14 G$ & $\begin{array}{l}\text { Length measure from the distal tip of } \\
\text { the glove's 2nd finger to the center } \\
\text { of the glove's } 1^{\text {st }} \text { finger crotch. } \\
\text { Surface measure. }\end{array}$ \\
\hline $\begin{array}{l}\text { Length measure from the glove's } \\
\text { distal tip of } 2^{\text {nd }} \text { to distal tip of the } \\
\text { glove's } 1^{\text {st }} \text { finger. Surface measure. }\end{array}$ \\
\hline 136
\end{tabular}

\subsection{Hand and glove measurements}

Once the measurements were defined for both the hand and the glove, the 3D scans and gloves were measured in centimeters using standard measurement tools. For all 3D scans, AnthroScan software from Human Solutions was used to extract measurements. First, landmarks were visually located on the 3D scans and a digital landmark was placed in the software program to create an accurate guide for measurements.

For glove measurements, product landmarks were placed using a permanent pen on the interior, and measurements were collected manually three times with a flexible, plastic tape measure. The measurements were averaged and documented in a spreadsheet.

\subsection{Size Selection}

Prior to analyzing measurements, ease assumptions were created for the glove to hand comparison. As there are no formal guidelines for incorporating ease values into gloves, ease assumptions were derived by the authors based on the assumptions of the glove use and fit. Because the glove is meant to be worn "next-to-skin" and excess material can hinder the athlete's performance, the researchers concluded that no ease should be incorporated into the hand measurement values.

Using the size selection process provided online by FootJoy, the measurements $4 \mathrm{H}$ (length of the distal tip of the $3^{\text {rd }}$ finger) and an additional circumference measurement at the knuckles were used to segment the subjects into the appropriate size category. A tape measure and a diagram were provided by the company online [15] to assist in finding the correct size (figure 4). The diagram uses a numerical code for each measurement of 0-9. Table 2 shows the FootJoy numerical code and the corresponding measurement used to compare the middle finger $(4 \mathrm{H})$ and the circumference of the hand. The subjects' measurements were translated into the FootJoy numerical code, and a size was selected based on the diagram in figure 5 . 

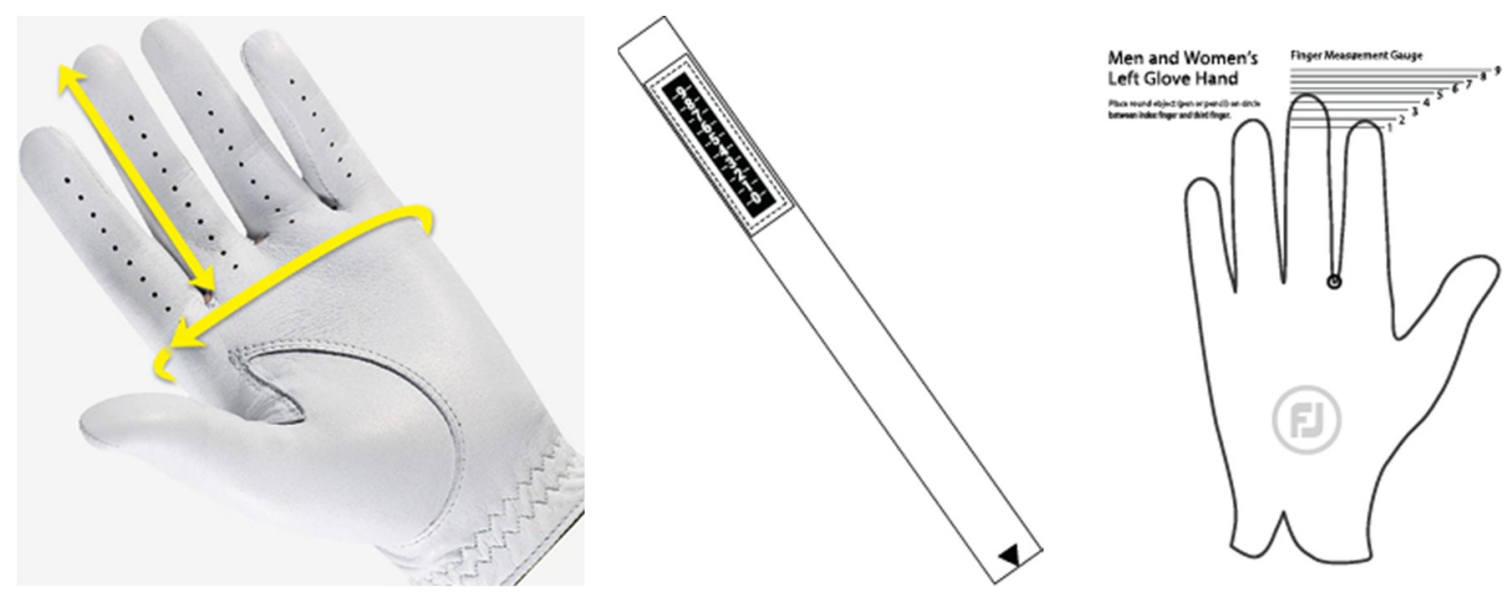

Fig. 4. FootJoy gloves size selection process

Table 2. FootJoy gloves size selection: Measurements of the hand compared to FootJoy numerical code for middle finger length and circumference of hand.

\begin{tabular}{|c|c|c|}
$\begin{array}{c}\text { FootJoy } \\
\text { Size } \\
\text { Selection }\end{array}$ & $\begin{array}{c}\text { Middle Finger } \\
\text { Length: } \mathbf{4 H}\end{array}$ & $\begin{array}{c}\text { Circumference of hand } \\
\text { around first knuckle } \\
\text { (excluding thumb): 8H }\end{array}$ \\
\hline 0 & - & 18.9 \\
\hline 1 & 7 & 19.5 \\
\hline 2 & 7.4 & 20.1 \\
\hline 3 & 7.8 & 20.7 \\
\hline 4 & 8.2 & 21.3 \\
\hline 5 & 8.5 & 21.9 \\
\hline 6 & 8.8 & 22.5 \\
\hline 7 & 9 & 23.1 \\
\hline 8 & 9.3 & 23.7 \\
\hline 9 & 9.6 & 24.3 \\
\hline
\end{tabular}

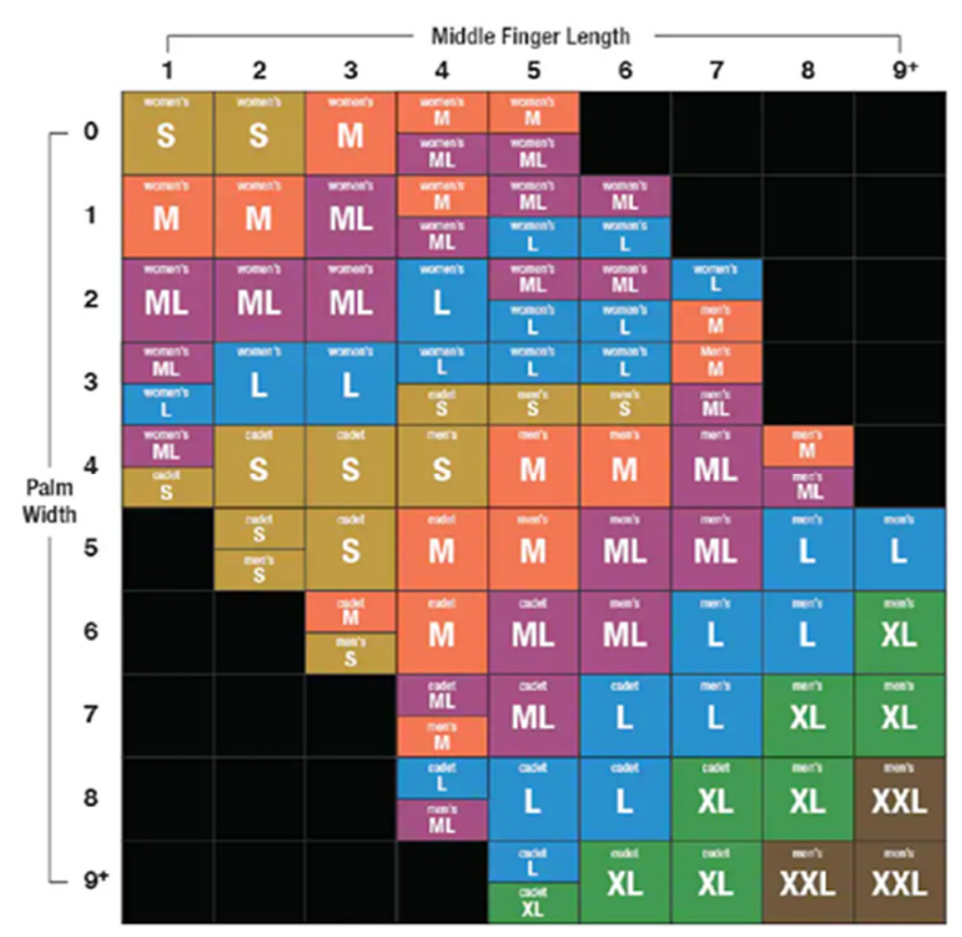

Fig. 5. FootJoy gloves size selection process 


\section{Results}

\subsection{Subjects}

3D hand scans from a convenience sample of thirty women, aged 18-65 years, from Oregon and Minnesota were collected for this study. Twenty-nine scans were useable.

\subsection{Hand anthropometric measurements}

Basic statistics were performed on the measurements on the subject data acquired from the 3D hand scans. For each of the fourteen measurements described in Table 3, the maximum (MAX), minimum (MIN), average, standard deviation, and range were calculated for the sample. Table 3 presents those measures.

Table 3. Hand anthropometric measurements (in centimeters).

\begin{tabular}{|c|c|c|c|c|c|c|c|c|c|c|c|c|c|c|}
\hline & 17 & $2 \mathrm{H}$ & $3 \mathrm{H}$ & $4 \mathrm{H}$ & $5 \mathrm{H}$ & $6 \mathrm{H}$ & $7 \mathrm{H}$ & $8 \mathrm{H}$ & $9 \mathrm{H}$ & $10 \mathrm{H}$ & $11 \mathrm{H}$ & $12 \mathrm{H}$ & $13 \mathrm{H}$ & $14 \mathrm{H}$ \\
\hline MAX & 15.51 & 7.64 & 9.09 & 9.65 & 9.29 & 7.10 & 14.00 & 23.86 & 23.39 & 11.90 & 21.18 & 18.19 & 12.44 & 20. \\
\hline IIN & 11.80 & 4.80 & 5.70 & 6.90 & 6.40 & 4.57 & 9.40 & 18.60 & 18.20 & 9.20 & 16.80 & 14.50 & 7.20 & 13.58 \\
\hline Avere & 13.76 & 5.90 & 7.24 & 7.99 & 7.22 & 5.89 & 11.60 & 20.95 & 20.11 & 10.78 & 18.70 & 16.22 & 10.43 & 16.43 \\
\hline Stanc & 1.00 & 0.66 & 0.79 & 0.63 & 0.68 & 0.53 & 1.11 & 1.22 & 1.08 & 0.70 & 1.13 & 1.04 & 1.07 & 1.31 \\
\hline ang & 3.71 & 2.84 & 3.39 & 2.75 & 2.89 & 2.53 & 4.60 & 5.26 & 5.19 & 2.70 & 4.38 & 3.69 & 5.24 & 6.9 \\
\hline
\end{tabular}

The sample used for this study had a broad range of hand sizes and variances. $6 \mathrm{H}$, a linear measurement of the distal tip of the $1^{\text {st }}$ finger to the interdigital fold, represented the smallest range and standard deviation of the sample, $2.53 \mathrm{~cm}$ and $.53 \mathrm{~cm}$ respectively. $14 \mathrm{H}$, a surface measurement from the distal tip of the $2^{\text {nd }}$ digit through the web-space to the distal tip of the $1^{\text {st }}$ finger, represented the largest range and standard deviation of the sample, $6.93 \mathrm{~cm}$ and $1.31 \mathrm{~cm}$ respectively.

\subsection{Glove specification measurements}

From specification data acquired from the size small (LS), medium (LM), medium-large (LML) and large (LL) leather golf gloves, averages were calculated for each of the fourteen identified measures. Table 4 presents those measures. Overall, the specifications for these gloves showed good linearity across each measurement between the sizes. This indicates that the manufacturing for these particular gloves is precise.

Table 4. Glove specification measurements (in centimeters).

\begin{tabular}{|c|c|c|c|c|c|c|c|c|c|c|c|c|c|c|}
\hline & $1 \mathrm{G}$ & $2 G$ & $3 G$ & $4 G$ & $5 G$ & $6 \mathrm{G}$ & $7 \mathrm{G}$ & $8 \mathrm{G}$ & $9 G$ & 10 & 11 & $12 \mathrm{G}$ & 13 & $14 \mathrm{G}$ \\
\hline$S$ & 15.03 & 6.5 & 8.26 & 8.4 & 7.7 & 5.2 & 13.2 & 19.6 & 20.73 & 9.53 & 17.8 & 15.23 & 11.06 & 17.76 \\
\hline LN & 16.06 & 6.6 & 8.43 & 8.8 & 7.83 & 5.56 & 3.16 & 19.7 & 19.06 & 10 & 18.56 & 15.1 & 11.4 & 17. \\
\hline & 16.33 & 6.9 & 8.63 & 9.05 & 8.23 & 6.13 & 13.76 & 20.93 & 19.76 & 10 & 18.96 & 16.46 & 12.16 & 8.8 \\
\hline Glove & 16.86 & 7.3 & 8.96 & 9.23 & 8.5 & 6.2 & 14.96 & 20.76 & 19 & 10.03 & 19.4 & 15.96 & 11.83 & 19. \\
\hline
\end{tabular}

\subsection{Size distribution based on glove fit system}

An analysis was conducted to understand how well the sample when divided into Small, Medium, Medium-Large, and Large sizes (based to FootJoy's size selection guide) fits into the women's glove models measured for the study. The size selection chart and directions (fig. 4, Table 2, and fig. 5) were used as a basis for analysis. Size distribution of the sample is found in figure 6 .

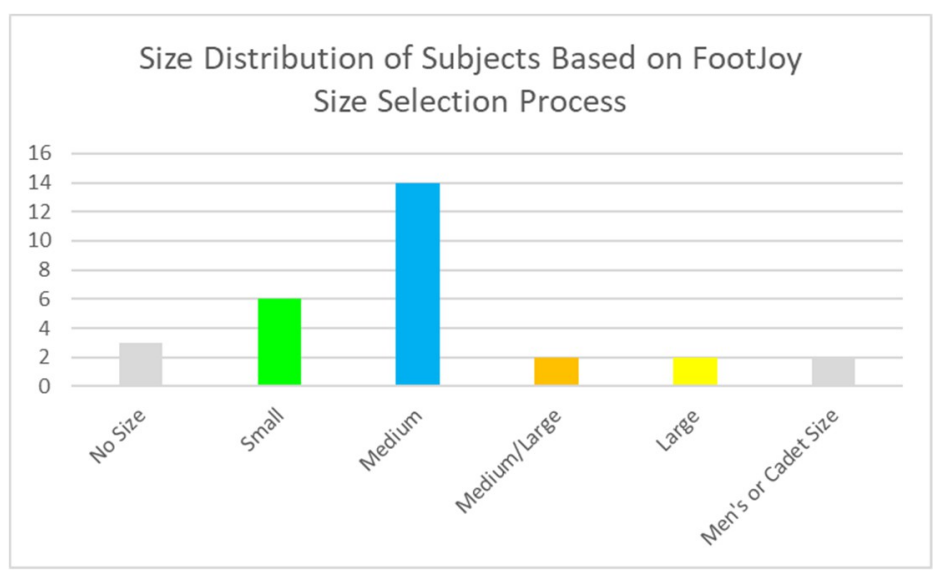

Fig. 6. Size distribution based on FootJoy size selection process 
Overall, nearly $83 \%$ of the sample fit into a women's specific glove. Twenty-four subjects fit into the women's FootJoy size system, with 2 subjects fitting in a Men's or Cadet Size and 3 subjects with no size recommendation. The women's medium size fit the most subjects $(n=14)$, followed by small $(n=6)$, medium/large (2), and large $(n=2)$.

\subsection{Hand anthropometric measures vs. glove specification measures}

Once the hand scans were assigned glove sizes, the researchers were able to compare the glove specifications to the 3D hand scan anthropometric measurements. Table 5 shows the results of the averaged glove specification measures compared to the average hand anthropometric measurements, for both sizes. The percentage of difference was calculated for each of the measures between glove specifications and the hand. If the resulting number was positive - then the glove was larger than the hand measure. If the resulting measure was negative, then the glove was smaller than the hand (too tight). In cases where the measurement was negative, it was highlighted in red in Table 5 as the consumer would not be able to properly fit into the glove. If the glove was more than $+15 \%$ different than the hand, it was highlighted in grey.

Table 5. Glove specification measures vs. hand anthropometric measurement data for Small, Medium, Medium-Large, and Large sizes (in centimeters).

\begin{tabular}{|c|c|c|c|c|c|c|c|c|c|c|c|c|c|c|}
\hline & $1 \mathrm{G} / \mathrm{H}$ & $2 \mathrm{G} / \mathrm{H}$ & $3 \mathrm{G} / \mathrm{H}$ & $4 \mathrm{G} / \mathrm{H}$ & $5 \mathrm{G} / \mathrm{H}$ & $6 \mathrm{G} / \mathrm{H}$ & $7 \mathrm{G} / \mathrm{H}$ & $8 \mathrm{G} / \mathrm{H}$ & $9 \mathrm{G} / \mathrm{H}$ & 10G/H & $11 \mathrm{G} / \mathrm{H}$ & $12 \mathrm{G} / \mathrm{H}$ & $13 \mathrm{G} / \mathrm{H}$ & 14G/H \\
\hline SMALL GLOVE & 15.03 & 6.5 & 8.26 & 8.4 & 7.7 & 5.2 & 13.2 & 19.6 & 20.73 & 9.53 & 17.8 & 15.23 & 11.06 & 17.76 \\
\hline SMALL & & & & & & & & & & & & & & \\
\hline SUBJECTS N=6 & 13.07 & 5.23 & 6.49 & 7.27 & 6.65 & 5.87 & 11.11 & 18.12 & 19.49 & 10.58 & 17.72 & 15.66 & 10.19 & 15.96 \\
\hline$\%$ Difference & $15.01 \%$ & $24.30 \%$ & $27.23 \%$ & $15.59 \%$ & $15.70 \%$ & $-11.41 \%$ & $18.80 \%$ & $8.19 \%$ & $6.38 \%$ & $-9.97 \%$ & $0.44 \%$ & $-2.74 \%$ & $8.49 \%$ & $11.26 \%$ \\
\hline MEDIUM GLOVE & 16.06 & 6.6 & 8.43 & 8.8 & 7.83 & 5.56 & 13.16 & 19.7 & 19.06 & 10 & 18.56 & 15.1 & 11.4 & 17.96 \\
\hline MEDIUM & & & & & & & & & & & & & & \\
\hline SUBJECTS $N=14$ & 13.46 & 6.02 & 7.35 & 7.99 & 7.23 & 5.77 & 11.32 & 18.70 & 20.04 & 10.63 & 18.62 & 16.07 & 10.41 & 16.46 \\
\hline$\%$ Difference & $19.28 \%$ & $9.67 \%$ & $14.75 \%$ & $10.10 \%$ & $8.36 \%$ & $-3.66 \%$ & $16.29 \%$ & $5.35 \%$ & $-4.87 \%$ & $-5.92 \%$ & $-0.34 \%$ & $-6.05 \%$ & $9.48 \%$ & $9.12 \%$ \\
\hline $\begin{array}{l}\text { MEDIUM-LARGE } \\
\text { GLOVE }\end{array}$ & 1633 & 6.90 & 8.63 & 905 & 8.23 & 6.13 & 13.76 & 20.93 & 1976 & 10.00 & 18.96 & 16.46 & 12.16 & 18.86 \\
\hline MEDIUM-LARGE & & & & & & & & & & & & & & \\
\hline SUBJECTS N=2 & 15.15 & 5.65 & 7.15 & 8.45 & 7.60 & 6.70 & 13.30 & 19.85 & 21.05 & 11.40 & 19.65 & 16.55 & 10.55 & 16.50 \\
\hline \% Difference & $7.79 \%$ & $22.12 \%$ & $20.70 \%$ & $7.10 \%$ & $8.29 \%$ & $-8.51 \%$ & $3.46 \%$ & $5.44 \%$ & $-6.13 \%$ & $-12.28 \%$ & $3.51 \%$ & $-0.54 \%$ & $15.26 \%$ & $14.30 \%$ \\
\hline LARGE GLOVE & 16.86 & 7.3 & 8.96 & 9.23 & 8.5 & 6.2 & 14.96 & 20.76 & 19 & 10.03 & 19.4 & 15.96 & 11.83 & 19.6 \\
\hline LARGE & & & & & & & & & & & & & & \\
\hline SUBJECTS N=2 & 13.85 & 5.45 & 6.60 & 7.85 & 6.70 & 5.35 & 12.10 & 20.60 & 20.10 & 10.85 & 18.60 & 16.90 & 9.85 & 15.25 \\
\hline$\%$ Difference & $21.73 \%$ & $33.94 \%$ & $35.76 \%$ & $17.58 \%$ & $26.87 \%$ & $15.89 \%$ & $23.64 \%$ & $0.78 \%$ & $7 \%$ & & $4.30 \%$ & & $20.10 \%$ & $28.52 \%$ \\
\hline
\end{tabular}

Results clearly show that glove specifications and hand anthropometric data are not aligned. Across all sizes, there are measurements that have a significant negative and positive percent difference between the glove and hand. This indicates that the proportion of the glove to hand is incorrect.

For the Small size comparison, measurements $6 \mathrm{G} / \mathrm{H}, 10 \mathrm{G} / \mathrm{H}$, and $12 \mathrm{G} / \mathrm{H}$ were all too small for the subjects, with measurement $6 \mathrm{G} / \mathrm{H}$ being $-11.41 \%$ too small. Measurements $1 \mathrm{G} / \mathrm{H}, 2 \mathrm{G} / \mathrm{H}, 3 \mathrm{G} / \mathrm{H}, 4 \mathrm{G} / \mathrm{H}$, $7 \mathrm{G} / \mathrm{H}$, and $14 \mathrm{G} / \mathrm{H}$ were all over $+10 \%$ between the hand and glove, meaning that the glove was over $10 \%$ larger than the hand sample measurements.

When comparing measurements for $1 \mathrm{G} / \mathrm{H}, 2 / \mathrm{GH}, 3 \mathrm{G} / \mathrm{H}, 4 \mathrm{G} / \mathrm{H}, 5 \mathrm{G} / \mathrm{H}, 7 \mathrm{G} / \mathrm{H}, 8 \mathrm{G} / \mathrm{H}, 12 \mathrm{G} / \mathrm{H}, 13 \mathrm{G} / \mathrm{H}$, and $14 \mathrm{G} / \mathrm{H}$, the Medium glove would be too large for the sample, up to $+19.28 \%$. Whereas, when comparing measurements for $6 \mathrm{G} / \mathrm{H}, 9 \mathrm{G} / \mathrm{H}, 10 \mathrm{G} / \mathrm{H}, 11 \mathrm{G} / \mathrm{H}$, and $12 \mathrm{G} / \mathrm{H}$, the Medium glove would be too small for the sample, up to $-5.92 \%$.

When comparing the Medium-Large glove, 1G/H, 2G/H, 4G/H, 5G/H, 7G/H, 8G/H, 13G/H, and 14G/H measurements were all too large for the sample subjects, up to $+22.12 \%$. Measurements $6 \mathrm{G} / \mathrm{H}, 9 \mathrm{G} / \mathrm{H}$, $10 \mathrm{G} / \mathrm{H}, 11 \mathrm{G} / \mathrm{H}$, and $12 \mathrm{G} / \mathrm{H}$ were all too small for the sample, up to $-12.28 \%$.

For the Large glove, measurements $1 \mathrm{G} / \mathrm{H}, 2 \mathrm{G} / \mathrm{H}, 2 \mathrm{G} / \mathrm{H}, 4 \mathrm{G} / \mathrm{H}, 5 \mathrm{G} / \mathrm{H}, 6 \mathrm{G} / \mathrm{H}, 7 \mathrm{G} / \mathrm{H}, 11 \mathrm{G} / \mathrm{H}, 13 \mathrm{G} / \mathrm{H}$, and $14 \mathrm{G} / \mathrm{H}$ were all too large compared to the hand, up to $+35.76 \%$.

This golf glove style is meant to provide a snug, next-to-skin fit so no ease was incorporated into the hand measurements for the comparison. Even if a 2-5\% ease value was added to the hand measurements, there would still be a significant measurement difference between the glove and hand. 


\section{Discussion and conclusion}

Results from this pilot study uncovered significant areas for improvement in terms of the glove to hand measurement relationship. Comparing product specifications to the actual sample demonstrated a clear need to improve the fit of the gloves for consumers, specifically the relationship between hand measurements and product specifications. Finger and palm length, as well as web-space areas offer significant opportunity for improvement. There is also a need for developing industry-wide ease standards. Standards should be based upon material thickness, flexibility, stretch, and glove functionality. Ease values should also be validated through wear testing.

This pilot study demonstrates one method of utilizing the data from 3D hand scan through comparing product specifications to hand measurements. Beyond product specific measurements, there is a need to develop new ways to apply 3D hand data to the design development of gloves and other products for the hand. Based upon the findings of this study, future research will be conducted to evaluate a larger sample of women, various glove brands/styles, and activities (e.g., firefighting, medical surgery and other sport).

\section{Acknowledgements}

This research was partially supported by the the Minnesota Agricultural Experiment Station, and the National Institute of Food and Agriculture, U.S. Department of Agriculture (Hatch under MIN-53-088), and the Office of the Vice President for Research and Innovation, University of Oregon.

\section{References}

[1] Professional Golf Association, "Fostering Women In Golf," PGA Impact. [Online]. Available: https://pgaimpact.org/fostering-women-in-golf.html. [Accessed: 1-Sep-2019].

[2] More Pars, "More Pars Golf Gifts - Golf Survival Guide." [Online]. Available: https://www.golfsurvivalguide.com/more-pars-golf-gifts/. [Accessed: 1-Sep-2019].

[3] S. Corriveau, "Applying Ergonomics to Industrial Glove Design." Occupational Health \& Safety (Waco, Tex.), vol. 83, no. 1, pp. 8, 10, 2014.

[4] N. Kim, R. Carufel, \& L. Griffin. "A Three-Dimensional Assessment of Hand Dynamics: A Pilot Study of Seven Hand Positions," $21^{\text {st }}$ Annual Applied Ergonomics Conference, Atlanta, GA, USA, March 26-29, 2018.

[5] L. Griffin, S. Sokolowski, \& E. Seifert. "Process Considerations in 3D Hand Anthropometric Data Collection." Ninth International Conference and Exhibition on 3D Body Scanning and Processing Technologies, Lugano, Switzerland, pp. 142-153, Online, Available: http://dx.doi.org/10.15221/18.121, October 16-17, 2018.

[6] Griffin, L., Sokolowski, S., Lee, H., Seifert, E., Kim, N., Carufel R. "Methods and Tools for 3D Measurement of Hands and Feet," In: Chung W., Shin C. (eds.) Advances in Interdisciplinary Practice in Industrial Design. AHFE 2018, vol. 790, pp. 49-58. Springer, Cham. Online, Available: https://doi.org/10.1007/978-3-319-94601-6 7, July 2018.

[7] L. Griffin, N. Kim, R. Carufel, S. Sokolowski, H. Lee, \& E. Seifert. "Dimensions of the Dynamic Hand: Implications for Glove Design, Fit, and Sizing," In: Chung W., Shin C. (eds.) Advances in Interdisciplinary Practice in Industrial Design. AHFE 2018, vol. 790, pp. 38-48, Springer, Cham, Online, Available: https://doi.org/10.1007/978-3-319-94601-6 6, July 2018.

[8] S.L. Sokolowski. "The Development of a Performance Hand Wear and Tools Product Innovation

Framework," Fashion and Textiles, accepted for publication in Fall 2019.

[9] E. Seifert, C. Curry, \& L. Griffin. "3D Anthropometric Assessment of Functional Hand Grasps for Surgeons and Medical Professionals." In 2019 Design of Medical Devices Conference 2019. American Society of Mechanical Engineers Digital Collection. Jul 19.

[10]S.L. Sokolowski \& C.T. Hoegsted. "The Application of the Performance Hand Wear and Tools Innovation Approach: Road Cycling Gloves." In: Shin C. (eds) Advances in Interdisciplinary Practice in Industrial Design. vol 968, pp. 105-111, Springer, Cham Online, Available: https://doi.org/10.1007/978-3-030-20470-9 13. 2019. 
[11]S.L. Sokolowski, L. Griffin, \& S. Chandrasekhar. "Current Technology Landscape for Collecting Hand Anthropometric Data," Ninth International Conference and Exhibition on 3D Body Scanning and Processing Technologies, Lugano, Switzerland, pp. 142-153, Online, Available: doi:10.15221/18.142, October 16-17, 2018.

[12]S. L. Sokolowski, \& L. Griffin. "Drawing Hands for Glove Design: Does the Data Match-Up?" In: Chung W., Shin C. (eds.) Advances in Interdisciplinary Practice in Industrial Design. AHFE 2018, vol. 790, pp. 68-77, Springer, Cham, Online, Available: https://doi.org/10.1007/978-3-31994601-6 9, July 2018.

[13] International Organization for Standardization. "ISO 7250-1: Basic Human Body Measurements for Technological Design - Part 1: Body Measurement Definitions and Landmarks," Geneva, Switzerland, 2017.

[14] "Statista - The Statistics Portal for Market Data, Market Research and Market Studies." [Online]. Available: https://www.statista.com/. [Accessed: 1-Sep-2019].

[15] "StaSof Women - FootJoy." [Online]. Available: https://www.footjoy.com/golf-gloves/women/stasofwomen/001STA.html?dwvar 001STA color=66784E-301\#start=2. [Accessed: 26-Sep-2019].

[16] "Golf Glove Fitting Guide | FootJoy." [Online]. Available: https://www.footjoy.com/fitting-mengloves.html. [Accessed: 1-Sep-2019]. 